\title{
Pharmacometric Characterization of Dabigatran Hemodialysis
}

\author{
Karl-Heinz Liesenfeld • Alexander Staab • \\ Sebastian Härtter · Stephan Formella $\cdot$ \\ Andreas Clemens • Thorsten Lehr
}

Published online: 26 March 2013

(C) The Author(s) 2013. This article is published with open access at Springerlink.com

\begin{abstract}
Background Hemodialysis has been shown to be a useful method of decreasing dabigatran plasma levels in situations that require rapid elimination of this thrombin inhibitor. However, there is currently no clinical recommendation for the accelerated/optimized elimination of dabigatran via hemodialysis (e.g., flow rates, filter type, duration of dialysis).

Objectives The primary objective of the present work was to characterize, via pharmacometric methods, the effects of different blood flow rates in hemodialysis on the pharmacokinetics of dabigatran, using data from a dedicated phase I dialysis study of end-stage renal disease (ESRD) patients. In addition, the effects of various clinically relevant hemodialysis settings were evaluated by simulation to assess their potential use in non-ESRD situations.

Methods Seven patients with ESRD were investigated in an open-label, fixed-sequence, two-period comparison trial. A population pharmacokinetic model was developed to fit the data and then used for various simulations. Data analyses were performed using NONMEM $^{\circledR}$, Berkeley Madonna, or SAS.
\end{abstract}

\footnotetext{
K.-H. Liesenfeld $(\bowtie) \cdot$ A. Staab · S. Härtter · T. Lehr

Boehringer Ingelheim Pharma GmbH \& Co. KG,

Birkendorfer Str. 65, 88397 Biberach, Germany

e-mail: karl-heinz.liesenfeld@ boehringer-ingelheim.com

S. Formella $\cdot$ A. Clemens

Boehringer Ingelheim Pharma GmbH \& Co. KG,

Ingelheim, Germany

T. Lehr

Saarland University, Saarbruecken, Germany
}

Results The pharmacokinetics of dabigatran were best described by a two-compartment model with first-order absorption and a lag time. In addition to total body clearance in ESRD subjects, a first-order dialysis clearance was implemented which was greater than zero during hemodialysis and zero during the interdialytic periods. The relationship between the dialysis clearance and the blood flow rate was best described by the Michaels function. Simulations showed that varying clinically relevant dialysis settings such as filter properties or flow rates had only minor effects. Dialysis duration had the strongest impact on dabigatran plasma concentration. The observed geometric mean redistribution effect after hemodialysis was low $(<16 \%)$. The final model was successfully evaluated through the prediction of plasma concentrations from a case report undergoing dialysis.

Conclusions This analysis allowed the influences of various hemodialysis parameters on the dabigatran plasma concentration to be predicted in detail for the first time. Dialysis duration was identified as having the strongest impact on the reduction in dabigatran plasma concentration. The model developed here can potentially serve as a tool to provide guidance when considering the use of hemodialysis in patients who have received dabigatran.

\section{Background}

Dabigatran is a novel synthetic, nonpeptidic, potent, specific, competitive, and reversible inhibitor of thrombin. Its marketed form is the prodrug dabigatran etexilate, which has been approved for the primary prevention of venous thromboembolism after total elective hip or knee replacement surgery, and for the prevention of stroke and systemic embolism in patients with nonvalvular atrial fibrillation 
(AF) in numerous countries. Further indications are under evaluation [1].

Dabigatran etexilate is rapidly absorbed and converted to dabigatran by esterase-catalyzed hydrolysis [2]. Maximum plasma concentrations of dabigatran occur approximately $2-3 \mathrm{~h}$ after oral dosing. The disposition is biexponential, the terminal half-life is $12-17 \mathrm{~h}[3,4]$, and steady state is attained after about 3 days of twice-daily treatment [5]. The total and peak systemic exposure are dose-proportional over the whole investigated range from $50 \mathrm{mg}$ up to $400 \mathrm{mg}$ [3]. Two food interaction studies showed that total areas under the plasma concentrationtime curve (AUC) and peak plasma concentrations $\left(C_{\max }\right)$ remained essentially unchanged under fasted and fed conditions. Food delayed the time to the $C_{\max }\left(t_{\max }\right)$ of dabigatran by about $2 \mathrm{~h}$ [6].

The oral bioavailability of dabigatran etexilate in capsules is about $6.5 \%$ [3]. Dabigatran is eliminated mainly (85\% of the dose) via renal glomerular filtration [2, 7-10], whereby $80 \%$ is excreted unchanged and $20 \%$ is eliminated as glucuronide. A protein binding study with radiolabeled dabigatran showed that $35 \%$ of the dabigatran is bound to plasma proteins over a wide concentration range [2]. The prodrug dabigatran etexilate-but not the active moiety dabigatran - is a substrate of P-glycoprotein (P-gp) [11].

Certain situations such as the need for emergency surgery in patients on anticoagulant medications may require rapid reversal of their anticoagulant effects. Hemodialysis has been shown to be one method that can reverse the anticoagulant effects of dabigatran by removing it from the blood [10, 12-14]. However, no investigation of various clinically relevant dialysis settings (such as filter properties or flow rates) aimed at a better understanding of the elimination of dabigatran has been carried out. One prior study was conducted in a subgroup of a phase I study, wherein six volunteers with end-stage renal disease (ESRD) were dialyzed after administration of $50 \mathrm{mg}$ dabigatran etexilate. It was shown that a substantial fraction of the dabigatran was eliminated by hemodialysis [10]. To further increase knowledge about dialyzing dabigatran, a dedicated phase I study was conducted in seven additional ESRD patients [12]. The study was designed using simulation methodology, with a focus on the maximization of potential redistribution effects [15]. The study demonstrated that a 4-h hemodialysis eliminated $49-59 \%$ of dabigatran from circulation [12].

The objectives of the work presented here were firstly to characterize the effects of hemodialysis at different blood flow rates on the pharmacokinetics of dabigatran by pharmacometric approaches using data from a dedicated phase I dialysis study [12]. The final model was evaluated through the prediction of external data [14]. Secondly, the effects of various clinically relevant hemodialysis conditions on dabigatran elimination were evaluated via simulations to assess their potential use in non-ESRD situations.

\section{Methods}

\subsection{Study Design}

The data from a phase I dialysis study performed by Khadzhynov and colleagues [12] served as the basis for our analysis. A detailed description of the study design and results was published previously [12]. In brief, the primary objectives of the study were the evaluation of blood and plasma dialysis clearance and the proportion of dabigatran removed from the central compartment during a standardized 4-h hemodialysis session. Secondary objectives were to evaluate pharmacokinetic measures, pharmacodynamics [activated partial thromboplastin time (aPTT) and the diluted thrombin time (dTT)], and safety parameters.

The study was designed as a nonrandomized, openlabel, two-period, fixed-sequence, multiple-dose study with two standard hemodialysis elimination settings (see below). A washout phase of $\geq 6$ weeks between periods was included. Seven dialysis-dependent ESRD subjects without atrial fibrillation were entered into and completed the trial. For both trial periods, three dabigatran etexilate doses were administered, each separated by a period of $21 \mathrm{~h}$ : one dose of $150 \mathrm{mg}$ dabigatran etexilate shortly after hemodialysis on day 1 ; one dose of $110 \mathrm{mg}$ dabigatran etexilate on day 2; and one dose of $75 \mathrm{mg}$ dabigatran etexilate $8 \mathrm{~h}$ before hemodialysis on day 3. Hemodialysis $4 \mathrm{~h}$ in duration was performed on days 1,3 , and 5 . The dialysis on day 1 was a standard dialysis without dabigatran exposure. On day 3, experimental dialysis was performed with different target blood flow rates of $200 \mathrm{~mL} /$ min and $400 \mathrm{~mL} / \mathrm{min}$ in the first and second periods, respectively. The dialysis on day 5 was a slightly modified standard dialysis with a blood flow rate of $300 \mathrm{~mL} / \mathrm{min}$ in both periods. The dialysate flow rate was $700 \mathrm{~mL} / \mathrm{min}$ during all dialysis periods. A large-surface-area, high-flux filter (Polyflux PF-210H dialyzer, Gambro Dialysatoren GmbH, Hechingen, Germany) was used. To allow subsequent comparisons with the results from various simulation scenarios, the dialysis settings from period 1 (blood flow rate of $200 \mathrm{~mL} / \mathrm{min}$ ) were used as reference data.

Dabigatran plasma concentrations were measured at the following planned time points in relation to the first dose of dabigatran etexilate in all subjects: $-4,0$ (day 1 , pre-dose samples), 21, 22, 23, 25, 27, 33 (day 2), 42, 43, 44, 46, 48, 50, 51, 52, 53, 54, 58 (day 3), 62, 70, 82 (day 4), 91, 95 (day 5) h. The hemodialysis times in relation to the first dose of dabigatran etexilate were -4 to $0 \mathrm{~h}$ on day 1,50 to 
$54 \mathrm{~h}$ on day 3 , and 91 to $95 \mathrm{~h}$ on day 5 . The study design, including the timing of samples in relation to the dabigatran dose administration and dialysis, is summarized in Fig. 1. Time of food intake in relation to administration varied from 17 to $90 \mathrm{~min}$ on the third day (fasted). Food intake time on day two was around $2 \mathrm{~h}$ before administration (fed).

To measure free and conjugated dabigatran, blood samples were drawn into EDTA-containing tubes and immediately placed in an ice/water bath $\left(0-4{ }^{\circ} \mathrm{C}\right)$ until centrifugation (at about $3,000 \times g$ for $10 \mathrm{~min}$ at $4{ }^{\circ} \mathrm{C}$ ) for plasma preparation. Plasma samples were stored at $-70{ }^{\circ} \mathrm{C}$ until analysis. The concentrations of nonconjugated, free dabigatran and total dabigatran (sum of free dabigatran + dabigatran glucuronide) in plasma were analyzed by highperformance liquid chromatography tandem mass spectrometry methods at Nuvisan GmbH (Neu-Ulm, Germany), as described elsewhere [10]. Accuracy ranged from -4.9 to $-2.6 \%$ for total dabigatran concentrations of 3.00-320 ng/ $\mathrm{mL}$. Precision for the same range was between 3.8 and $4.5 \%$. The lower limit of quantification was $1.00 \mathrm{ng} / \mathrm{mL}$. The method was validated according to the current Food and Drug Administration guidance on bioanalytical method validation [16].

\subsection{Data Analysis}

All modeling and simulation analyses were performed using the NONMEM $^{\circledR}$ software package (version VI 2.0; ICON Development Solutions, Ellicott City, MD, USA) or Berkeley Madonna (version 8.0.4; Berkeley Madonna Inc., University of California, Berkeley, CA, USA). Model selection was based on several criteria, such as goodness-offit plots, precision of model parameter estimates, and changes in the NONMEM $^{\circledR}$ objective function $-2 \log$ likelihood. When models were classified as nested, one model was declared superior to the other model when the objective function value was reduced by $3.84(P<0.05$, $1 d f)$. The first-order conditional estimation with interaction method was used throughout model development. SAS (version 9.2; SAS Institute Inc., Cary, NC, USA) was used for statistical analyses. Graphics were generated using SigmaPlot (version 10.0; Systat Software, Inc., Richmond, CA, USA) or Visio (Version 2007; Microsoft Corporation, Redmond, WA, USA).

The pharmacokinetic model that was developed to optimize the study design of the hemodialysis study served as a starting point for the data analysis [15]. The model consisted of two disposition compartments with first-order absorption and a lag time. An apparent dabigatran dialysis clearance $\left(\mathrm{CL}_{\text {dialysis }} / F\right)$ was implemented in addition to the intrinsic total body clearance $(\mathrm{CL} / F)$ in ESRD subjects (renal and nonrenal elimination). The dialysis clearance was greater than zero during the hemodialysis and zero during the interdialytic periods. The total apparent dabigatran clearance $\left(\mathrm{CL}_{\text {total }} / F\right)$ is the sum of $\mathrm{CL} / F$ and $\mathrm{CL}_{\text {dialysis }} / F$. The apparent dialysis clearances in $\mathrm{L} / \mathrm{h}$ were converted to actual dialysis clearances in $\mathrm{mL} / \mathrm{min}$, assuming a typical bioavailability of $6 \%$.

Initially, for each investigated blood flow rate, a separate apparent dabigatran dialysis clearance was estimated. In a second step, the relationship between blood flow rate and apparent dabigatran dialysis clearance was explored using various functions, such as a linear model, a maximum effect $\left(E_{\max }\right)$ model, and the Michaels equation (Eq. 1) [17]:

$\mathrm{CL}_{\text {dialysis }} / F=\frac{\operatorname{BFR}\left(\mathrm{e}^{\frac{K_{\circ} A}{\mathrm{BFR}} \cdot\left(1-\frac{\mathrm{BFR}}{\mathrm{DFR}}\right)}-1\right)}{\mathrm{e}^{\frac{K_{\circ} A}{\mathrm{BFR}} \cdot\left(1-\frac{\mathrm{BFR}}{\mathrm{DFR}}\right)}-\frac{\mathrm{BFR}}{\mathrm{DFR}}}$

where $K_{\mathrm{o}} A$ is the mass transfer-area coefficient for dabigatran of the dialysis filter used, DFR is the dialysate flow rate, and BFR is the blood flow rate.

Several statistical models to describe variability were evaluated. Residual variability was described by a proportional model. Interindividual variability (IIV) and interoccasion variability (IOV) were modeled using exponential random effect models. The following definitions of "occasion" were tested: (a) study period and (b) dosing interval.
Fig. 1 Schematic representation of the study design for a single period. $P K$ pharmacokinetic

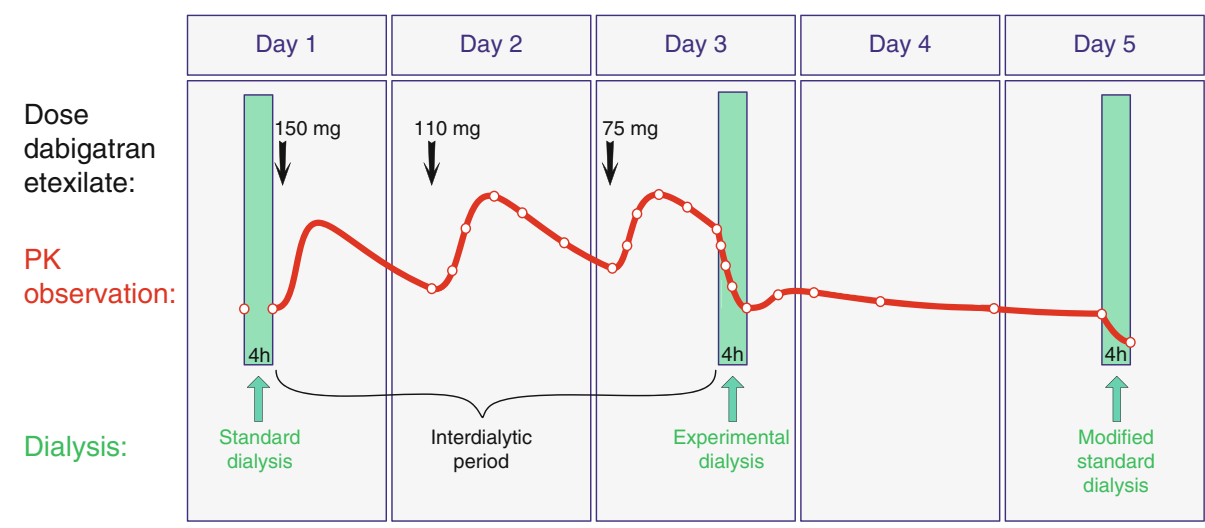


The maximum redistribution in percent (Redist ${ }_{\max }$ ) was calculated according to Eq. 2:

Redist $_{\max }=\frac{C_{\max } \text { after dialysis }-C_{\text {end dialysis }}}{C_{\text {end }} \text { dialysis }} \times 100 \%$

where $C_{\max }$ after dialysis is the maximum plasma concentration measured up to $16 \mathrm{~h}$ after dialysis was stopped, and $C_{\text {end }}$ dialysis is the plasma concentration at the end of dialysis.

\subsection{Simulations}

Three different clinical scenarios were simulated. Scenario 1 evaluated the effect of variations in dialysis settings on the reduction in plasma concentration. Simulated patients were given oral dabigatran and dialyzed as defined in the study protocol during period 1 [12]. Variations in the hemodialysis filter with respect to the manufacturer (Fresenius instead of Gambro), the effective membrane surface (reduced by $20 \%$ ), the membrane type (low-flux instead of high-flux membrane), and the membrane material (Poracton instead of Polyamix) were mimicked by varying the dialysis clearance by $-5,-10,-20$, and $+10 \%$, respectively. A reduced dialysate flow rate of $500 \mathrm{~mL} / \mathrm{min}$ instead of $700 \mathrm{~mL} / \mathrm{min}$ was calculated to reduce $\mathrm{CL}_{\text {dialysis }}$ by $5 \%$. Also, blood flow rates of 150,250 , and $400 \mathrm{~mL} / \mathrm{min}$ were simulated. In addition, a worst case scenario was investigated where several effects were added simultaneously, resulting in a reduction in the dialysis clearance of $40 \%$.

Scenario 2 systematically explored the maximum redistribution effect. Scenario 3 studied the effect of variations in the renal function and the duration of dialysis on the reduction in plasma concentration. In both scenarios, simulated AF patients were dosed with $150 \mathrm{mg}$ twice daily to steady state. Dialysis was started $12 \mathrm{~h}$ after the last dose. The duration of dialysis was varied from 1 to $10 \mathrm{~h}$. Creatinine clearance $\left(\mathrm{CL}_{\mathrm{CR}}\right)$ values of $30,50,70$, and $100 \mathrm{~mL} /$ min were simulated.

To mimic AF patients, all simulations were performed utilizing pharmacokinetic parameter estimates from the RE-LY trial [9]. Demographic and other covariates were set to a typical patient from the RE-LY trial (Caucasian male, 72 years old, body weight of $80.3 \mathrm{~kg}$, hemoglobin $14.3 \mathrm{mg} / \mathrm{dL}$, no comedications). Only median profiles were simulated.

\subsection{Model Evaluation}

The population pharmacokinetic model was evaluated by predicting the plasma concentration time course of an external data set from a patient undergoing hemodialysis from a literature report [14]. The structural dialysis model developed in this report was applied. The pharmacokinetic absorption and distribution parameters from a typical AF patient in the RE-LY trial [9] were used. The total body clearance was set to $10 \mathrm{~L} / \mathrm{h}$. The apparent dialysis clearance was calculated to be $180.3 \mathrm{~L} / \mathrm{h}$, based on the reported average blood flow rate of $320 \mathrm{~mL} / \mathrm{min}$. The dialysis duration was $6 \mathrm{~h}$, as reported. Interindividual variability in bioavailability $(F)$ was set to zero. An additional interindividual variability of $15 \%$ was introduced for the blood flow rate, as this was reported to vary [14]. Interindividual variability of $10 \%$ in the mass transfer-area coefficient $\left(K_{\mathrm{o}} A\right)$ was introduced to reflect the uncertainty in its value. The residual variability from the RE-LY trial was applied. An appropriate dose was developed to reach the reported plasma concentration of $76 \mathrm{ng} / \mathrm{mL}$ at the start of hemodialysis $48 \mathrm{~h}$ after the last dosing. The same filter and dialysis flow rate were applied in the present study as used in the case reported [14]. The plasma concentration time profile of the patient was simulated 5,000 times and the corresponding median and 5th and 95th percentiles were calculated. To evaluate the predictive performance, the predicted profile was overlaid with the reported concentrations of the patient from the publication [14].

\section{Results}

\subsection{Population Pharmacokinetic Model}

All 7 ESRD subjects enrolled in this study were white males with a mean age of 38.3 years (range 27-53 years) and a mean body weight of $74.0 \mathrm{~kg}$ (range 60-87 kg). The dataset consisted of 308 observations (total dabigatran plasma concentrations) and 42 dose administration records. All data records were included in the analysis. The geometric means of the trough plasma concentrations after the second dose were $140 \mathrm{ng} / \mathrm{mL}$ (coefficient of variation [CV] $54.2 \%$ ) and $128 \mathrm{ng} / \mathrm{mL}$ (CV $44.5 \%$ ) in periods 1 and 2, respectively.

The pharmacokinetics of dabigatran were best described using a two-compartment disposition model with first-order absorption. As the timing of food intake relative to dabigatran administration varied throughout the study, different lag times and an $E_{\max }$ function of the food intake time relative to dabigatran administration were incorporated into the model to account for this effect on the absorption.

The final pharmacokinetic model included two parallel first-order clearance pathways. First, an apparent total body dabigatran clearance $(\mathrm{CL} / F)$ was introduced. $\mathrm{CL} / F$ in ESRD subjects was estimated at $12.4 \mathrm{~L} / \mathrm{h}$. Variations in the serum creatinine levels did not explain any variation in the total body drug clearance. Second, an apparent dialysis clearance $\left(\mathrm{CL}_{\text {dialysis }} / F\right)$ was implemented in parallel to describe the accelerated drug clearance caused by 
hemodialysis. The dialysis clearance was greater than zero during the hemodialysis periods and zero during the interdialytic periods. The effect of blood flow rate on the dialysis clearance was best described using the Michaels equation [17]; the objective function decreased significantly by 22.354 points compared to the model with constant dialysis clearance.

$K_{\mathrm{o}} A$ was estimated at $313 \mathrm{~mL} / \mathrm{min}$. Figure 2 depicts the schematic pharmacokinetic model and the relationship between blood flow rate and dialysis clearance. The total apparent dabigatran clearance $\left(\mathrm{CL}_{\text {total }} / F\right)$ is the sum of $\mathrm{CL} /$ $F$ and $\mathrm{CL}_{\text {dialysis }} / F$. Model-derived values of the different apparent clearance parameters for the investigated blood flow rates are shown in Table 1.

Interindividual variability was implemented in $\mathrm{CL} / F$ and the apparent volume distribution of the central compartment $\left(\mathrm{V}_{2} / F\right)$. Interoccasion variability was implemented in $F$ and the first-order absorption rate constant $\left(k_{\mathrm{a}}\right)$, where one occasion was defined for each dosing interval. The residual variability was described by a proportional residual variability model. All parameters of the final model were estimated with good precision (relative standard errors ranging from 4.6 to $48.5 \%$, Table 2). The goodnessof-fit plots of the final model are shown in Fig. 3. All observed versus predicted concentrations were in general spread randomly around the line of identity, indicating that the data were well described by the model; no trend over time was observable. The epsilon shrinkage was $10.4 \%$.

\subsection{Simulations}

The effects of varying the dialysis settings are shown in Fig. 4. Compared to the reference, the effects of individual factors on the reduction in plasma concentration were rather small $(-7$ to $+8 \%)$. Even in a worst case scenario where the effects accumulated, only a limited decrease in plasma concentration of $14 \%$ compared to the reference was predicted.

Simulation scenario 2 systematically investigated the maximum redistribution effect. Simulations predicted a maximum redistribution effect in $\mathrm{AF}$ patients with a low creatinine clearance of $30 \mathrm{~mL} / \mathrm{min}$ after a 10 -h dialysis (Fig. 5). The maximum typical redistribution was $26 \%$, which corresponds to an increase in dabigatran plasma concentration of $8.5 \mathrm{ng} / \mathrm{mL}$. After shorter dialysis duration of $4 \mathrm{~h}$, the typical redistribution would be even smaller, with an increase of $1.2 \mathrm{ng} / \mathrm{mL}$.

The effects of dialysis duration and total body clearance on the relative reduction in plasma concentration are shown in Fig. 6a. The impact of dialysis duration on the absolute reduction in dabigatran plasma concentration is shown in Fig. 6b. Three different starting concentrations are shown as examples, reflecting the observed 50th, 90th, and 99.6th percentiles of the trough plasma concentration of the

Table 1 Apparent total body clearance during the interdialytic period $(\mathrm{CL} / F)$, apparent dialysis clearance $\left(\mathrm{CL}_{\text {dialysis }} / F\right)$, and apparent total clearance $\left(\mathrm{CL}_{\text {total }} / F\right)$, as derived from the parameters of the final population pharmacokinetic model

\begin{tabular}{llll}
\hline $\begin{array}{l}\text { Blood flow rate } \\
(\mathrm{mL} / \mathrm{min})\end{array}$ & \multicolumn{3}{l}{ Apparent clearance parameters } \\
\cline { 2 - 4 } & $\begin{array}{l}\mathrm{CL} / F \\
(\mathrm{~L} / \mathrm{h})\end{array}$ & $\begin{array}{l}\mathrm{CL}_{\text {dialysis }} / F \\
(\mathrm{~L} / \mathrm{h})\end{array}$ & $\begin{array}{l}\mathrm{CL}_{\text {total }} / F \\
(\mathrm{~L} / \mathrm{h})\end{array}$ \\
\hline 200 & 12.4 & 148.5 & 160.9 \\
300 & 12.4 & 176.4 & 188.8 \\
400 & 12.4 & 192.7 & 205.1 \\
\hline
\end{tabular}

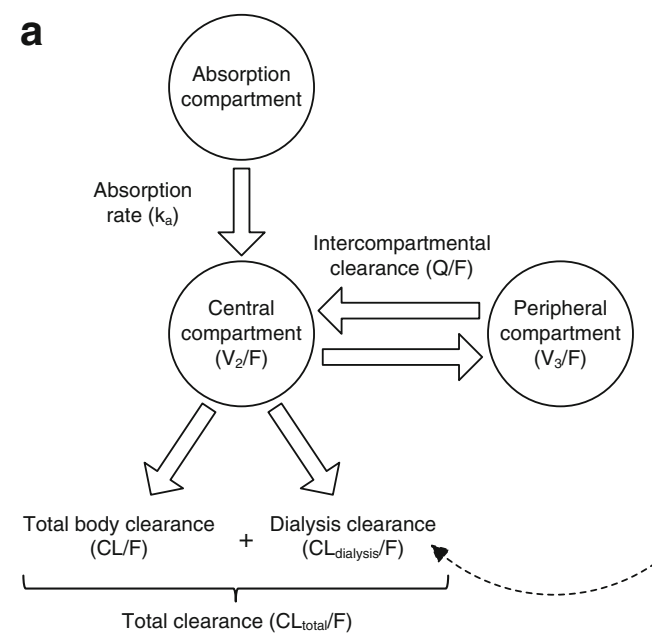

b

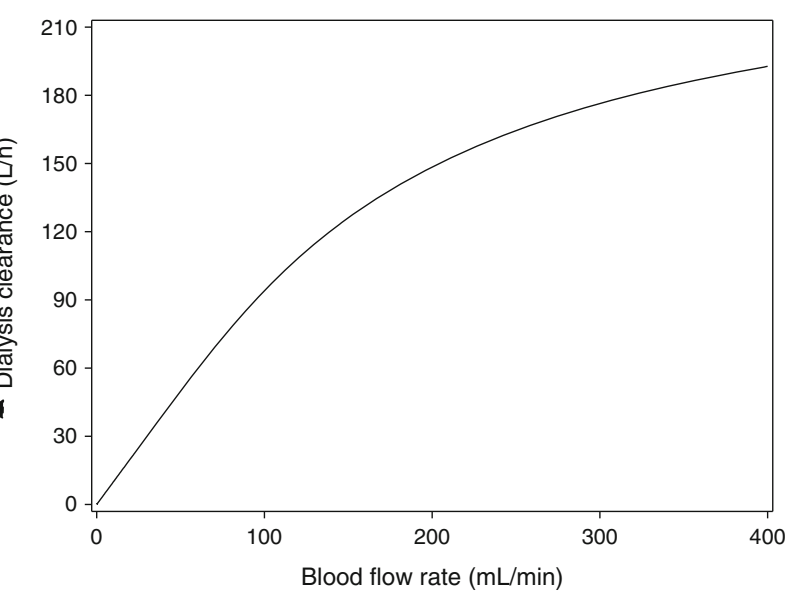

Fig. 2 a Schematic illustration of the final pharmacokinetic model. b Relationship between blood flow rate and apparent dialysis clearance of dabigatran for a dialysate flow rate of $700 \mathrm{~mL} / \mathrm{min}$ and an estimated mass transfer-area coefficient of $313 \mathrm{~mL} / \mathrm{min}$ 
Table 2 Parameter estimates from the final population pharmacokinetic model

\begin{tabular}{|c|c|c|c|}
\hline Parameter & Value & RSE $(\%)$ & Description \\
\hline \multicolumn{4}{|l|}{ Fixed effects } \\
\hline $\mathrm{CL} / F^{\mathrm{a}}(\mathrm{L} / \mathrm{h})$ & 12.4 & 28.71 & Total body clearance (renal and nonrenal) \\
\hline$V_{2} / F(\mathrm{~L})$ & 531 & 22.60 & Volume of distribution of the central compartment \\
\hline$Q / F(\mathrm{~L} / \mathrm{h})$ & 152 & 14.34 & Intercompartmental clearance \\
\hline$V_{3} / F(\mathrm{~L})$ & 499 & 9.42 & Volume of distribution of the peripheral compartment \\
\hline$k_{\mathrm{a}}\left(\mathrm{h}^{-1}\right)$ & 0.821 & 16.81 & First-order absorption rate constant \\
\hline ALAG (h) & 1.67 & 4.56 & Absorption lag time \\
\hline $\mathrm{ALAG}_{3} 3 \mathrm{rd}(\mathrm{h})$ & $0^{\mathrm{b}}$ & - & Absorption lag time of the third dose (fasted) \\
\hline$F$ & $1.00^{\mathrm{b}}$ & - & Relative bioavailability \\
\hline $\mathrm{EC}_{50 \text { food time }}{ }^{\mathrm{c}}(\mathrm{h})$ & 0.556 & 11.13 & $\begin{array}{l}\text { Time between dose administration and food intake at which the } \\
\text { effect on bioavailability is half of the maximum effect }\end{array}$ \\
\hline$F_{\min \text { food time }}{ }^{\mathrm{c}}$ & $0^{\mathrm{b}}$ & - & $\begin{array}{l}\text { Minimum bioavailability when time between dose administration } \\
\text { and food intake is } 0 \text { (fixed to } 0 \text { due to limited data) }\end{array}$ \\
\hline Hill $_{\text {food time }}{ }^{\mathrm{c}}$ & 6.10 & 48.52 & $\begin{array}{l}\text { Hill factor describing the steepness of the relation between time to } \\
\text { food intake and the relative bioavailability }\end{array}$ \\
\hline$K_{\mathrm{o}} A^{\mathrm{d}}(\mathrm{mL} / \mathrm{min})$ & 313 & 23.39 & Hemodialyzer mass transfer-area coefficient \\
\hline
\end{tabular}

Random effects: interindividual variability (IIV) and interoccasion variability (IOV)

$\begin{array}{llll}\text { IIV CL/F }(\mathrm{CV} \%) & 40.4 & 43.01 & \text { IIV in the total body clearance } \\ \text { IIV } V_{2} / F(\mathrm{CV} \%) & 14.3 & 43.07 & \begin{array}{l}\text { IIV in the apparent volume of distribution of the central } \\ \text { compartment }\end{array} \\ \text { IOV } k_{\mathrm{a}}(\mathrm{CV} \%) & 64.0 & 30.24 & \text { IOV in the relative first-order absorption rate constant } \\ \text { IOV } F(\mathrm{CV} \%) & 48.0 & 26.91 & \text { IOV in the relative bioavailability }\end{array}$

Random effects: residual variability

$\begin{array}{llll}\mathrm{PRV}(\mathrm{CV} \%) & 8.5 & 24.00 & \text { Proportional residual variability }\end{array}$

${ }^{\mathrm{a}} \mathrm{CL}_{\text {total }} / F=\mathrm{CL}_{\text {dialysis }} / F+\theta_{\mathrm{CL} / F} \times \mathrm{e}^{\eta \mathrm{CL}}$

b Parameters fixed

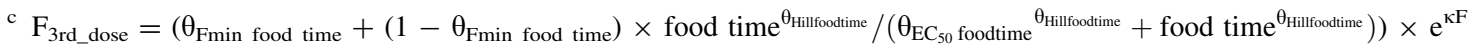

d See Eq. 1

$C V$ coefficient of variation, $R S E$ relative standard error, $C L_{\text {total }} / F$ total apparent dabigatran clearance, $C L_{\text {dialysis }} / F$ apparent dabigatran dialysis clearance, $\theta$ symbol for fixed-effect parameter estimate, $\eta$ symbol for interindividual variability, $F_{3 r d \_d o s e}$ relative bioavailability of the third dose in each period, $\kappa$ symbol for interoccasion variability

$150 \mathrm{mg}$ dose group in the RE-LY trial [18]. A substantial reduction in plasma concentration was predicted that was also dependent on the dialysis duration. The effect of the total body clearance on the reduction in plasma concentration was rather small over the time range investigated ( $<7 \%$ difference). The impact on the reduction in the plasma concentration was strong during the first few hours, but the response curves became shallower the longer the dialysis continued.

\subsection{Model Evaluation}

The final population pharmacokinetic model was externally evaluated through the prediction of the time profile of the plasma concentrations measured in a patient undergoing hemodialysis [14]. The predictions showed good agreement with the measured values; all observed concentrations were within the $90 \%$ prediction interval (Fig. 7).

\section{Discussion}

A population pharmacokinetic model was successfully developed for ESRD subjects to characterize the effects of hemodialysis at different blood flow rates on the plasma concentrations of dabigatran. The highly variable absorption process was clearly separated from the hemodialysis process. This allowed precise estimates to be obtained for the hemodialysis parameters and an acceptable description of the redistribution process.

In this ESRD population, a low total body dabigatran clearance of $12.4 \mathrm{~L} / \mathrm{h}$ was estimated. Considering the negligible renal clearance of dabigatran in this population $(<0.04 \mathrm{~L} / \mathrm{h})$ [12], it can be concluded that the estimated total body clearance mainly covers nonrenal excretion. In the RE-LY study, a total dabigatran clearance of $69.6 \mathrm{~L} / \mathrm{h}$ was reported for typical AF patients [9]. This total clearance is a composite of renal and nonrenal elimination 


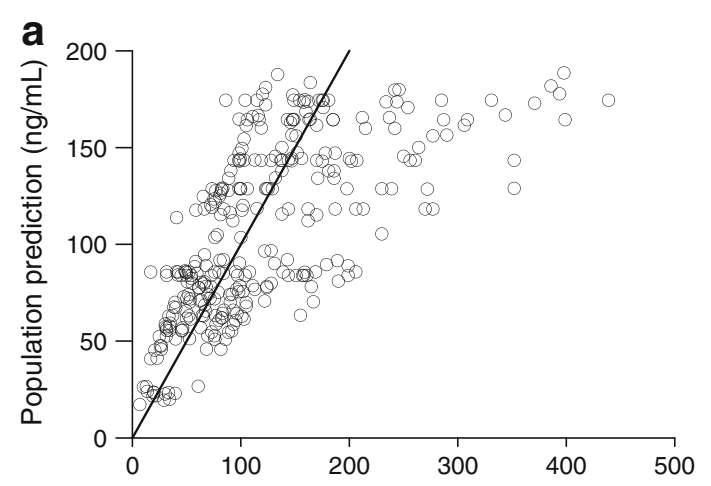

Observed dabigatran plasma concentration $(\mathrm{ng} / \mathrm{mL})$

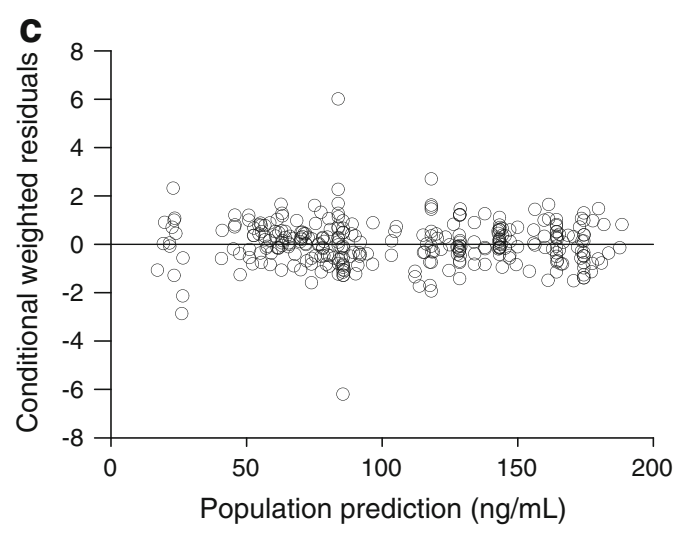

Fig. 3 Goodness-of-fit plots of the final population pharmacokinetic model. Observed plasma concentrations vs. population predictions (a) and individual predictions (b). Solid lines indicate lines of
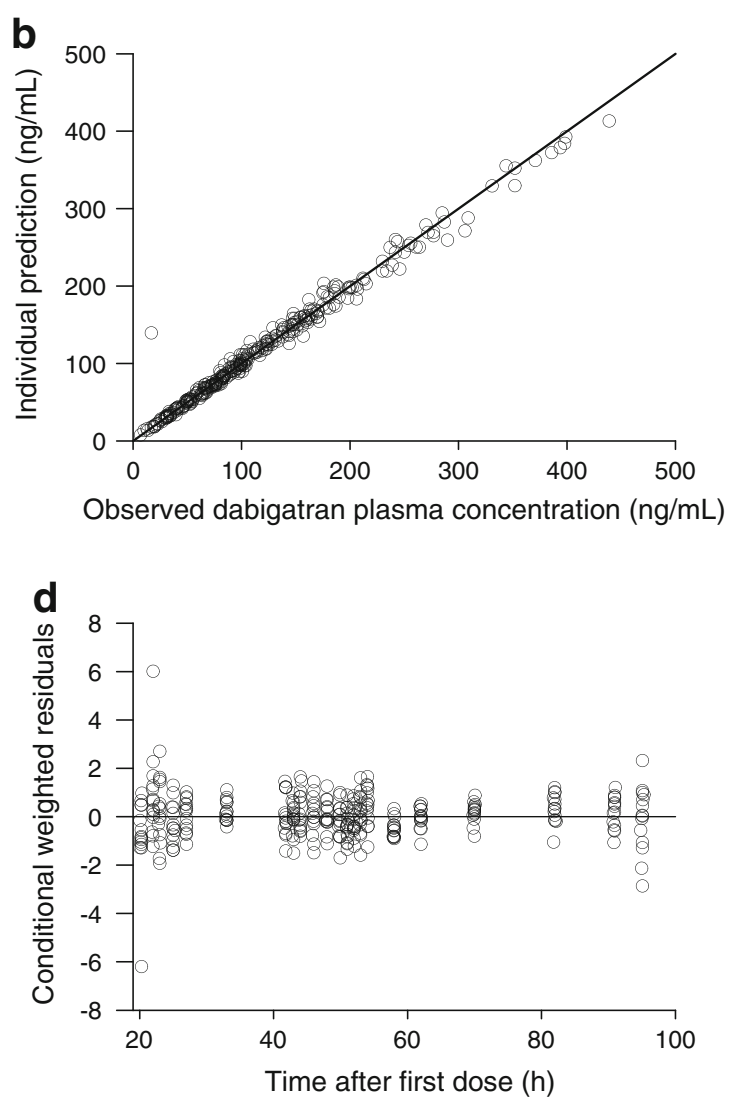

identity. Conditional weighted residuals vs. population predictions (c) and time after first dose (d)
Fig. 4 Simulated percentage changes in dialysis clearance and the resulting changes in reduction in plasma concentration for various dialysis settings compared to the reference. Reference settings were: blood flow rate $(\mathrm{BFR})=200 \mathrm{~mL} / \mathrm{min}$, dialysate flow rate $($ DFR $)=700 \mathrm{~mL} / \mathrm{min}$, filter $=$ large surface, high flux (Gambro PF-210H). For details, see "Methods"

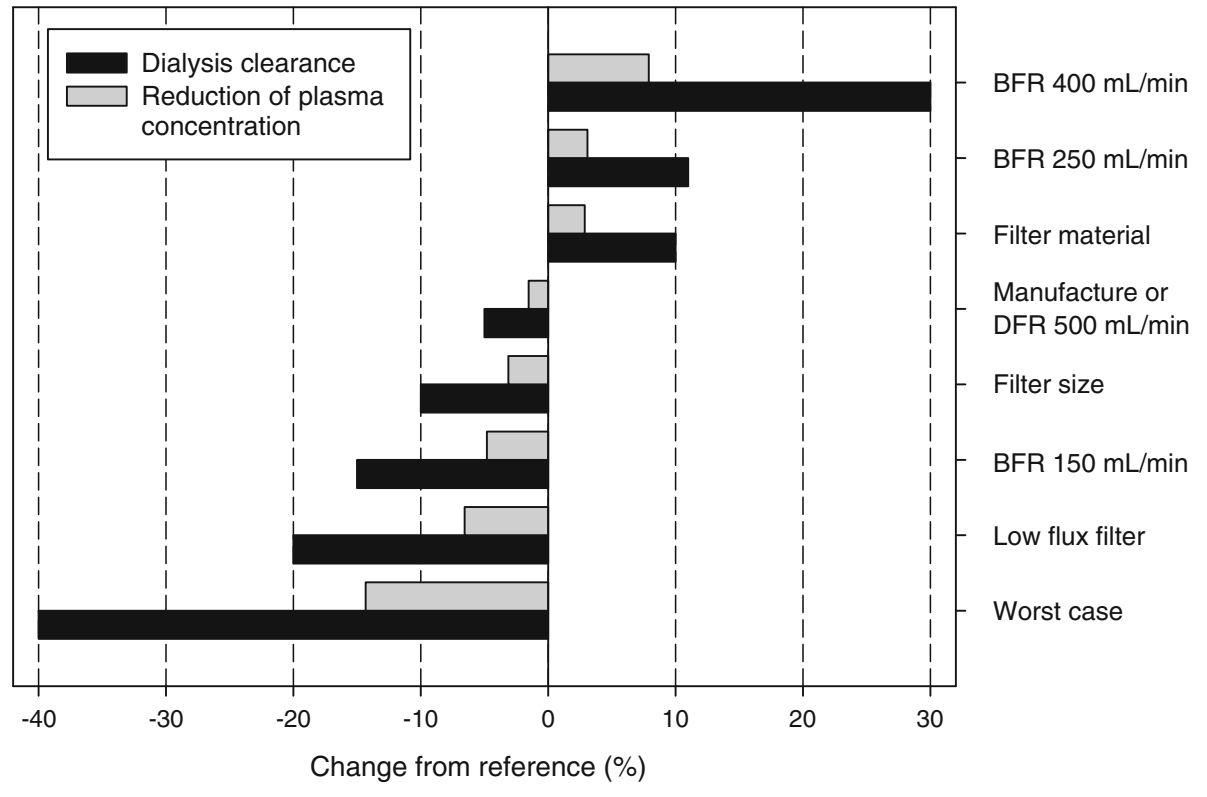

pathways. Assuming that the nonrenal clearance component calculated from the present analysis is applicable, then the contribution of the renal elimination to the total clearance for typical AF patients is $82 \%$. This agrees well with results from a healthy volunteers in a phase I study in which about $85 \%$ of the dabigatran dose was eliminated renally in non-ESRD subjects [2]. The absorption and distribution processes of dabigatran in ESRD patients were 


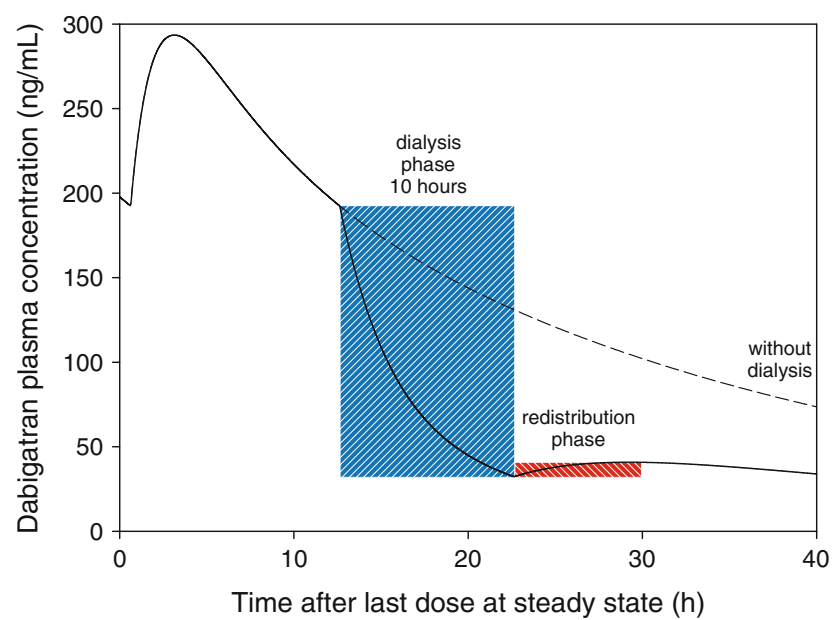

Fig. 5 Simulated time profile of plasma concentration for the maximum redistribution effect observed after a 10-h dialysis in a patient with a creatinine clearance of $30 \mathrm{~mL} / \mathrm{min}$ (solid line) and without dialysis (dotted line)

comparable to those in healthy subjects [7]. The 21-h dosing interval during the study caused variations in food intake on days 2 and 3 in relation to dabigatran administration. To account for this difference, different lag times and an $E_{\max }$ function were included in the model. The effect observed on $t_{\max }$ was absolutely identical to that noted in a report in the literature [6]. Even though the food effect was statistically significant, the described effect needs to be interpreted with caution due to the specific population, the specific study design and the small sample size.

The relationship between the blood flow rate, the dialysis flow rate and the dialysis clearance was best described by the well-established Michaels model [17]. This is not surprising, as the equation was specifically developed to quantify dialysis and has been frequently applied [19]. The equation provides great flexibility in predicting the effects of variations in the dialysis flow rate and the blood flow rate. Applied to the present data, it clearly demonstrates the limitations of increased flow rates, since doubling the blood flow rate from 200 to $400 \mathrm{~mL} / \mathrm{min}$ increases the dialysis clearance by only $30 \%$ and results in an additional reduction of the dabigatran concentration by only about $8 \%$. The Michaels model assumes a constant mass transfer-area coefficient. This coefficient is a property of the solute and the dialyzer. $K_{\mathrm{o}} A$ is expected to be independent of solute concentrations and flow rates. Even though some publications report different findings [20-22], the differences in $K_{\mathrm{o}} A$ reported were small, so the assumption seems acceptable. The simulations demonstrated the flexibility and usefulness of the applied Michaels model, since changes in the blood flow rate and dialysate flow rate can be directly translated into changes in $\mathrm{CL}_{\text {dialysis }} / F$.
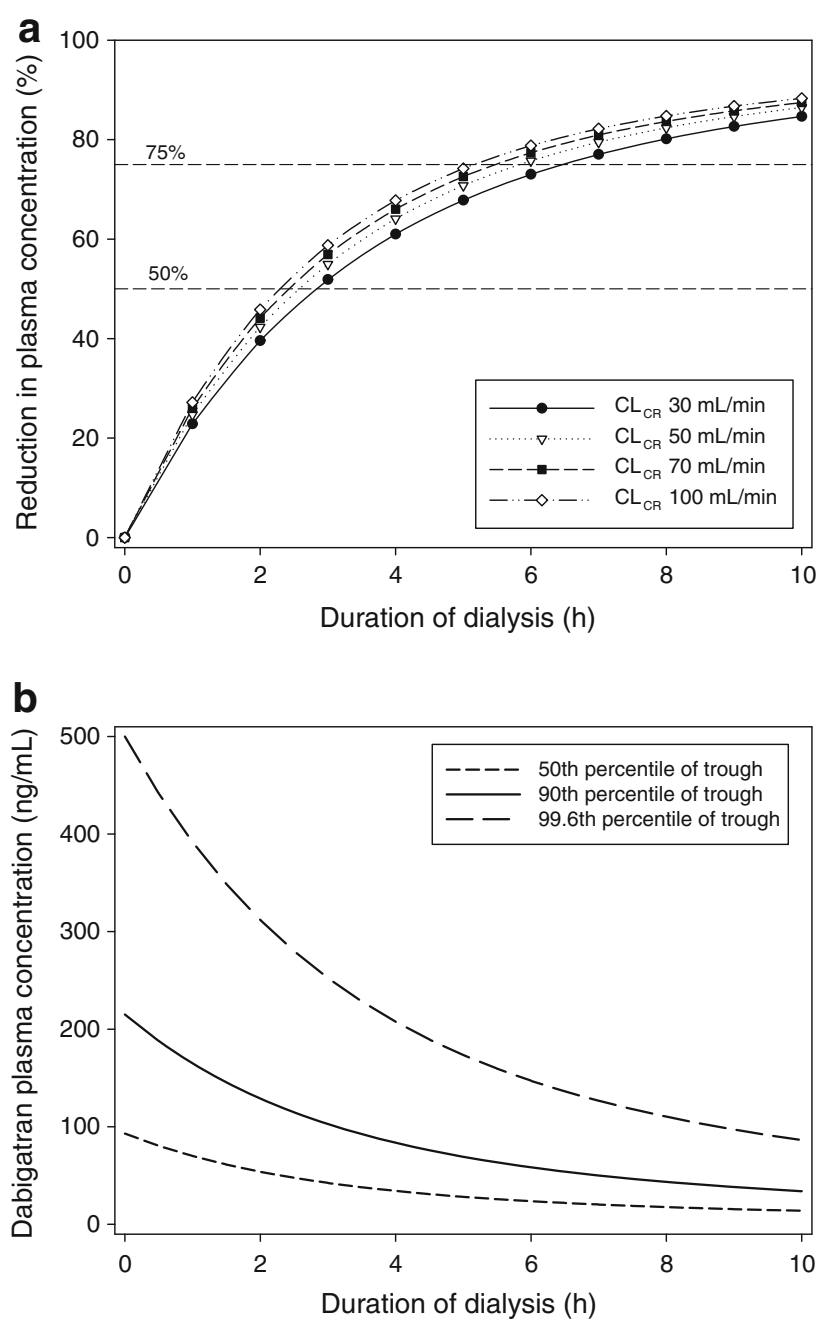

Fig. 6 a Predicted reduction in plasma concentration (\%) vs. duration of dialysis displayed for patients with different creatinine clearance values. b Predicted plasma concentrations vs. duration of dialysis for three different initial concentrations representing the observed median and the 90th and 99.6th percentiles of the trough plasma concentrations of the $150 \mathrm{mg}$ dose group in the RE-LY trial. $C L_{C R}$ creatinine clearance

Overall, no interindividual variability was identified in the dialysis clearance. This finding is in agreement with the low variability observed in the extraction ratio $(<10 \%$ geometric coefficient of variation) in the previous phase I study [12].

Overall, the simulations revealed that variations in dialysis settings have only a limited effect on the reduction in plasma concentration, whereas the dialysis duration is a key factor in the reduction process. Due to the first-order elimination process, the reduction in the plasma concentration is initially large, but it becomes smaller with increasing dialysis duration.

As redistribution of dabigatran after the end of the hemodialysis may result in a clinically relevant increase in 


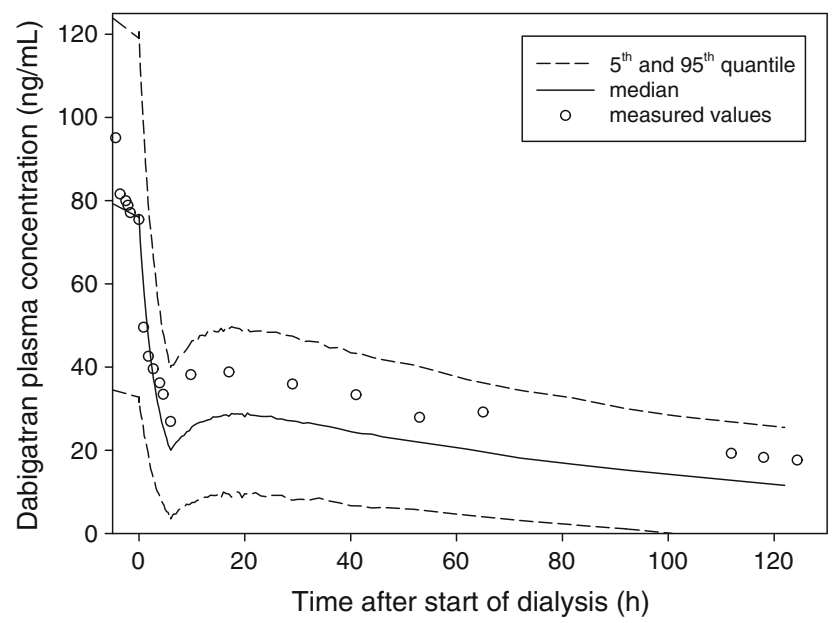

Fig. 7 External prediction of the time profile of the plasma concentrations measured in a patient undergoing hemodialysis. Measured values are shown as open circles. Predicted median and 5th and 95th percentiles are represented as lines

plasma concentration, the study design was optimized for a maximum redistribution effect [15]. In general, the extent of redistribution depends on several factors, such as the amount of drug available in peripheral compartments for redistribution, the intercompartmental clearance, the intrinsic clearance, and the hemodialysis clearance. The simulations for dabigatran showed that the average redistribution effect after dialysis is probably low. This finding is in agreement with the observed study results, which showed a small geometric mean redistribution of $\leq 15.5 \%$ [12].

The effect of renal function on the elimination of dabigatran is well characterized and expected for a drug that is mainly eliminated via the kidney [10]. In previous analyses, the relationship between $\mathrm{CL}_{\mathrm{CR}}$ and dabigatran clearance was described by a hockey-stick or an $E_{\max }$ model [7, 9]. In the analysis presented here, no relationship could be established between dabigatran clearance and the serum creatinine or $\mathrm{CL}_{\mathrm{CR}}$ measurements of the ESRD subjects. This is not surprising, as renal elimination in ESRD patients does not play any role [12].

Even though the renal function plays a major role in the excretion of dabigatran in non-ESRD patients, simulations predicted that the renal status of the dialyzed patient may be of minor importance with respect to the reduction in the plasma concentration and the redistribution effect (Fig. 6a). This is not surprising, since $\mathrm{CL}_{\text {dialysis }} / F$ is approximately 2to 3-fold higher than the body clearance of an $\mathrm{AF}$ patient with median renal function, and therefore represents the major elimination pathway during the dialysis.

The final model was evaluated through the prediction of measured plasma concentrations from a case report of a dabigatran-treated patient undergoing dialysis [14]. The almost negligible decline in the terminal phase confirms the assumed low total body clearance of this patient. The observed profile was well within the $90 \%$ prediction interval and underlines the predictive performance of the model. However, although this single case was predicted successfully, additional data would be required to fully validate the predictive performance of the model.

The early application of pharmacometric approaches during the design phase of the study contributed significantly to its success [12]. The primary focus during the optimization of the study design was on the dosing scheme, the start time, and the duration of the dialysis. The aim was to reach comparable plasma concentrations to those in $\mathrm{AF}$ patients in order to be able to assess the redistribution effect in subjects with therapeutic dabigatran plasma concentrations and to allow precise estimation of the dialysis parameters. The developed dosage regimen of $150 \mathrm{mg}$, $110 \mathrm{mg}$, and $75 \mathrm{mg}$ administered during the interdialytic period resulted in plasma concentrations in the range of the expected values (predicted $C_{\max }: 154 \mathrm{ng} / \mathrm{mL}$; observed $C_{\text {max }}: 176 \mathrm{ng} / \mathrm{mL}$ for period 1 and $159 \mathrm{ng} / \mathrm{mL}$ for period 2).

The analysis presented here has some limitations. First, the number of study subjects was relatively small $(n=7)$, with a total of 28 hemodialysis sessions. Nevertheless, all parameters were estimated precisely, and the absorption and distribution processes of dabigatran in ESRD patients were comparable to those in healthy subjects [7]. Secondly, all study subjects were male Caucasians in the therapeutic concentration range. However, there is no evidence that the pharmacokinetics of dabigatran are significantly influenced by ethnicity or sex after adjustment for renal function [7, 9]. Furthermore, since the dialysis clearance is concentration independent and mainly influenced by certain physicochemical properties of the dialysis filter and the protein binding of the studied drug, it seems justified to expect that results would be similar in women, patients of other ethnicities, and importantly in patients with the target indication, AF. Also, similar results would theoretically be expected in patients with supratherapeutic plasma concentrations.

\section{Conclusions}

Overall, the effect of dialysis is a complex interplay of many factors, such as duration of dialysis, start of dialysis, blood flow rate, dosing history, patient demographics, and many others. This first thorough analysis of the effects of various dialysis factors on dabigatran pharmacokinetics may allow the effect of dialysis on the plasma concentration to be predicted under various conditions. Dialysis duration was identified as having the greatest impact on the extent of dabigatran plasma concentration reduction. The developed model can potentially serve as a tool to provide guidance when considering the use of hemodialysis in 
patients that have received dabigatran. Further data will be required to validate the predictive performance of the model.

Acknowledgments This analysis was supported by Boehringer Ingelheim Pharma GmbH \& Co. KG. The authors wish to thank P. Tanswell for helpful editorial support in the preparation of this manuscript.

Conflict of interest All authors are employees of Boehringer Ingelheim Pharma GmbH \& Co. KG. The authors have no other conflicts of interest that are directly related to the content of this study.

Open Access This article is distributed under the terms of the Creative Commons Attribution Noncommercial License which permits any noncommercial use, distribution, and reproduction in any medium, provided the original author(s) and the source are credited.

\section{References}

1. Van de Werf F, Brueckmann M, Connolly SJ, et al. A comparison of dabigatran etexilate with warfarin in patients with mechanical heart valves: The Randomized, phase II study to Evaluate the sAfety and pharmacokinetics of oraL dabIGatran etexilate in patients after heart valve replacemeNt (RE-ALIGN). Am Heart J. 2012;163:931-7.

2. Blech S, Ebner T, Ludwig-Schwellinger E, et al. The metabolism and disposition of the oral direct thrombin inhibitor, dabigatran, in humans. Drug Metab Dispos. 2008;36:386-99.

3. Stangier J, Rathgen K, Stähle H, et al. The pharmacokinetics, pharmacodynamics and tolerability of dabigatran etexilate, a new oral direct thrombin inhibitor, in healthy male subjects. Br J Clin Pharmacol. 2007;64:292-303.

4. Stangier J, Stähle H, Rathgen K, et al. Pharmacokinetics and pharmacodynamics of the direct oral thrombin inhibitor dabigatran in healthy elderly subjects. Clin Pharmacokinet. 2008;47:47-59.

5. Clemens A, Haertter S, Friedman J, et al. Twice daily dosing of dabigatran for stroke prevention in atrial fibrillation - a pharmacokinetic justification. Curr Med Res Opin. 2012;28:195-201.

6. Stangier J, Stahle H, Rathgen K. Effect of food and pantoprazole on the bioavailability of the direct thrombin inhibitor dabigatran in healthy subjects. J Thromb Haemost. 2005;3.

7. Dansirikul C, Lehr T, Liesenfeld K-H, et al. A combined pharmacometric analysis of dabigatran etexilate in healthy volunteers and patients with atrial fibrillation or undergoing orthopaedic surgery. Thromb Haemost. 2012;107:775-85.

8. Lehr T, Haertter S, Liesenfeld K-H, et al. Dabigatran etexilate in atrial fibrillation patients with severe renal impairment: dose identification using pharmacokinetic modeling and simulation. J Clin Pharmacol. 2011;52:1373-8.

9. Liesenfeld K-H, Lehr T, Dansirikul C, et al. Population pharmacokinetic analysis of the oral thrombin inhibitor dabigatran etexilate in patients with non-valvular atrial fibrillation from the RE-LY trial. J Thromb Haemost. 2011;9:2168-75.

10. Stangier J, Rathgen K, Stähle $\mathrm{H}$, et al. Influence of renal impairment on the pharmacokinetics and pharmacodynamics of oral dabigatran etexilate: an open-label, parallel-group, singlecentre study. Clin Pharmacokinet. 2010;49:259-68.

11. Stangier J, Stähle H, Rathgen K, et al. Pharmacokinetics and pharmacodynamics of dabigatran etexilate, an oral direct thrombin inhibitor, with coadministration of digoxin. J Clin Pharmacol. 2012;52:243-50.

12. Khadzhynov D, Wagner F, Clemens A, et al. Effective elimination of dabigatran by haemodialysis: A phase I single-centre study in patients with end-stage renal disease. Thromb Haemost. 2013 Feb 7;109(4). [Epub ahead of print]

13. Wanek MR, Horn ET, Elapavaluru S, et al. Safe use of hemodialysis for dabigatran removal before cardiac surgery. Ann Pharmacother. 2012;46:e21.

14. Warkentin TE, Margetts P, Connolly SJ, et al. Recombinant factor VIIa (rFVIIa) and hemodialysis to manage massive dabigatran-associated postcardiac surgery bleeding. Blood. 2012; 119:2172-4.

15. Liesenfeld K-H, Lehr T, Moschetti V, Formella S, Clemens A, Staab A, et al. Modelling and simulation to optimize the study design investigating the haemodialysis of dabigatran in patients with end stage renal disease (ESRD) [abstract]. PAGE 20 (2011). http://www.page-meeting.org/?abstract=2001.

16. Food and Drug Administration. Guidance for industry: bioanalytical method validation. Silver Spring: Food and Drug Administration, 2001.

17. Michaels AS. Operating parameters and performance criteria for hemodialyzers and other membrane-separation devices. Trans Am Soc Artif Intern Organs. 1966;12:387-92.

18. Connolly SJ, Ezekowitz MD, Yusuf S, et al. Dabigatran versus warfarin in patients with atrial fibrillation. $\mathrm{N}$ Engl $\mathrm{J}$ Med. 2009;361:1139-51.

19. Shinaberger JH. Quantitation of dialysis: historical perspective. Semin Dial. 2001;14:238-45.

20. Allen R, Frost TH, Hoenich NA. The influence of the dialysate flow rate on hollow fiber hemodialyzer performance. Artif Organs. 1995; 19:1176-80.

21. Leypoldt JK, Cheung AK, Agodoa LY, et al. Hemodialyzer mass transfer-area coefficients for urea increase at high dialysate flow rates. Kidney Int. 1997;51:2013-7.

22. Ouseph R, Ward RA. Increasing dialysate flow rate increases dialyzer urea mass transfer-area coefficients during clinical use. J Am Soc Nephrol. 1999;10:199A. 